\title{
Meeting report \\ 8th International Conference: Primary Therapy of Early Breast Cancer, St Gallen, Switzerland, March 12-15 2003
}

Hilary Glen and Robert J Jones

\author{
Beatson Oncology Centre, Western Infirmary of Glasgow, Glasgow, UK \\ Corresponding author: Robert J Jones (e-mail: R.Jones@beatson.gla.ac.uk) \\ Published: 30 May 2003 \\ Breast Cancer Res 2003, 5:198-201 (DOI 10.1186/bcr611) \\ (C) 2003 BioMed Central Ltd (Print ISSN 1465-5411; Online ISSN 1465-542X)
}

\begin{abstract}
The International St Gallen Breast Cancer Conference concentrates almost exclusively on adjuvant, multimodal primary therapy for early breast cancer. Begun 25 years ago, this meeting was initially held every 4 years, but therapeutic progress, new strategies and provocative trials data have accelerated to the extent that conferences are now held biennially. The meeting this year was attended by almost 3000 delegates. Major topics included new prognostic and predictive markers in early breast cancer, the best use of adjuvant chemotherapy and endocrine therapy, and innovations in local surgery and radiation therapy.
\end{abstract}

Keywords: adjuvant, chemotherapy, endocrine, predictive, prognostic

\section{Introduction}

The tone of the 3-day meeting was set by Umberto Veronesi (European Institute of Oncology, Milan, Italy) at the opening session. The ongoing aim of primary therapy in early breast cancer is moving from the concept of 'maximum tolerated treatment' to that of 'minimum necessary treatment'. Toward this goal, the St Gallen consensus continues to strive to define a group of patients with exceptionally good prognosis who require minimal primary therapy, and much of the conference was concerned with how to improve this definition, as well as how best to treat the groups at higher risk for subsequent relapse. Throughout the meeting a recurrent theme was the distinction between prognostic information (which defines a level of risk) and predictive information (which predicts response to a particular therapy).

The St Gallen 2003 consensus statement is still being written and will be published in the summer (in the Journal of Clinical Oncology), and therefore we will not discuss its likely content, but we review some of the key issues discussed at the plenary and poster sessions.

\section{New prognostic and predictive markers in early breast cancer}

Daniel F Hayes (University of Michigan, Ann Arbor, USA) introduced the concept of positive predictive power as a means of assessing the validity of a predictive marker. Martine Piccart (Jules Bordet Institute, Brussels, Belgium) summarized promising new markers that may have prognostic and predictive value in the management of early breast cancer. These included uPA/Pal-1, cyclin E and cDNA microarrays, but all of these require further evaluation in prospective trials. Stephen Braun (Universitätsklinikum, Innsbruck, Austria) presented data suggesting that immunocytological demonstration of bone marrow micrometastases has independent prognostic value, and prospective evaluation of this technique aimed at ascertaining its predictive value is being planned. 


\section{Adjuvant hormone therapy}

The results of a large trial comparing the adjuvant use of anastrazole and tamoxifen in combination have been discussed elsewhere [1], but many speakers speculated that aromatase inhibitors are likely to play an increasing role in the future adjuvant treatment of breast cancer. In particular, Kathleen Pritchard (Sunnybrook Regional Cancer Centre, Toronto, Canada) discussed the need for further studies examining their role in the treatment of endocrine receptor positive, Her2 positive breast cancer. This was supported by laboratory data from Kent Osborne (Baylor College of Medicine, Houston, TX, USA). He presented compelling laboratory evidence that forced high level expression of Her2 in breast cancer cells stimulated the agonist activity of tamoxifen in the nucleus in a manner dependent upon activation of tyrosine kinase cascades. Particularly fascinating was his observation that this agonist activity of tamoxifen was reversed by the epidermal growth factor receptor tyrosine kinase inhibitor Iressa (gefitinib, ZD1839, AstraZeneca, Alderley Park, Cheshire, UK). These data offer significant new insight into crosstalk between growth factor receptor pathways and steroid receptors, and point toward possible strategies for therapeutic manipulation of tamoxifen resistance pathways.

Stefan Aebi (Inselspital, Institut für Medizinische Onkologie, Bern, Switzerland) and Pritchard both raised the issue of optimization of adjuvant endocrine therapy and the role of ovarian function suppression in young, premenopausal women. Two new trials from the International Breast Cancer Study Group (TEXT [Tamoxifen and Exemestane Trial] and SOFT [Suppression of Ovarian Function Trial] [2]) will address this issue.

\section{How best to combine endocrine and chemotherapy}

Probably the most assertive new data presented at the meeting came from Kathy Albain (Loyola University Medical Centre, Chicago, IL, USA), who presented the 10-year update from the North American Intergroup trial 0100. This was a three-arm study comparing tamoxifen alone with tamoxifen commenced at the same time as CAF (cyclophosphamide, doxorubicin, 5-FU) chemotherapy with tamoxifen commenced upon completion of the same chemotherapy [3]. This confirmed previous overview conclusions that tamoxifen confers a marked disadvantage in disease-free survival (DFS) and overall survival (OS) if administered during chemotherapy rather than on completion. However, Osborne warned that this effect might not be the same for other forms of oestrogen manipulation such as ovarian suppression or aromatase inhibition.

The issue of whether optimal endocrine therapy should complement or substitute for chemotherapy still provoked some debate, and this issue will be addressed in the forthcoming International Breast Cancer Study Group trial (PERCHE [Premenopausal Endocrine Responsive Chemotherapy trial]) [2].

\section{Advances in adjuvant chemotherapy}

Piccart described mixed findings to date from the first adjuvant trials using taxane-based chemotherapy regimens. Although initial findings suggested statistically significant differences in both DFS and OS with the addition of a taxane, perhaps most notably in the US Intergroup CALGB 9344 study [4], these early trials are largely confounded by the perceived suboptimal efficacy of the control treatments when compared with optimal anthracycline-containing regimens.

Although many of the taxane trials will not report for some time, reports from two large European anthracycline trials are expected at the Annual Meeting of the American Society for Clinical Oncology this year (Chicago, IL, 31 May 2003). Encouragingly, the French Adjuvant Study Group 05 (previously reported by Citron and coworkers [5]) presented the 10-year update on a trial comparing two doses of FEC (5-fluorouracil, epirubicin, cyclophosphamide) in the treatment of poor prognosis patients. This demonstrated ongoing DFS and OS advantage of patients treated at the higher dose.

Dose density, as opposed to total dose, may be an important variable in the context of adjuvant chemotherapy. Larry Norton (Memorial-Sloan Kettering Cancer Centre, New York, USA) outlined the rationale for this. Piccart reviewed the recent findings of the CALGB 9741 trial [6], which demonstrated a marked benefit from increased dose density without altering the total amount and type of drugs used. This is clearly an area to be addressed in future trials.

\section{Issues in radiotherapy}

Intra-operative electron therapy is a new approach currently undergoing trial evaluation in Milan and this was described by Roberto Orecchia (European Institute of Oncology, Milan, Italy). Oscar Streeter (Norris Cancer Centre, Los Angeles, CA, USA) described a North American brachytherapy approach in which sealed sources are introduced into a balloon placed intraoperatively in the tumour cavity following conservative surgery. Such an approach of partial breast irradiation is based on the assumption that the great majority of local relapses occur in the affected quadrant. However, Harry Bartelink (Netherlands Cancer Institute, Amsterdam, The Netherlands) cautioned against its application outwith clinical trials, citing Danish data that challenged this assumption.

Bartelinck also challenged the practice of restricting postmastectomy radiotherapy to patients with heavy nodal involvement. The Danish data suggested that these patients gain relatively less from radiotherapy than do patients with lighter or no nodal involvement. 


\begin{abstract}
Pathology
With the plethora of new prognostic and predictive tests currently under evaluation, it is essential that assays and techniques are reliable and standardized between centres. Barry Gusterson (University of Glasgow, Glasgow, UK) reminded us that there is still no consensus on the definition (or importance) of lymph node micrometastasis, and Giuseppe Viale (European Institute of Oncology, Milan, Italy) highlighted the ongoing issues of quality assurance. These issues have important consequences for the planning and interpretation of multicentre trials, and the introduction of new technologies such as microarrays will present new challenges in this regard.
\end{abstract}

Viale discussed special histological subtypes with prognostic importance, in particular ductal carcinoma with extensive necrosis/fibrosis, which carries a particularly poor prognosis even in the absence of lymph node metastasis.

\section{Surgical issues}

It is clear that sentinel lymph node biopsy is becoming the standard of care in many large centres. The emphasis is on this being a purely diagnostic procedure, and William Wood (Emory University Hospital, Atlanta, GA, USA) described his own practice in which patients undergo sentinel lymph node biopsy before definitive surgery in order that all the relevant prognostic and staging information can be obtained before planning therapy. This allows careful multidisciplinary evaluation and optimizes the use of preoperative systemic therapy. Although in his centre this practice enabled better cosmetic results and did not have a deleterious effect on outcome, no data have demonstrated superior survival after preoperative chemotherapy. Marco Colleoni (European Institute of Oncology, Milan, Italy) pointed out that the increased use of preoperative chemotherapy is of considerable interest, because this would enable the rapid assessment of predictive factors at an individual patient level rather than at a trial population level.

Wood and Viviana Galimberti (European Institute of Oncology, Milan, Italy) both discussed the value of biopsy of the internal mammary node as part of the sentinel node procedure because this 'upstages' cancer in around 10\% of cases.

\section{New drugs}

José Baselga (Hospital Universitario Vall d'Hebron, Barcelona, Spain) discussed existing and new approaches to growth factor signalling pathways. In particular he discussed $2 \mathrm{C} 4$, a monoclonal antibody to Her2 that has the potential to block promitotic signalling pathways even in those cancers that do not over-express this receptor. V Craig Jordan (Northwestern University Medical School, Chicago, IL, USA) described further potential for new agents in the oestrogen pathway, but also outlined an interesting rationale for cyclic use of agonists, antagonists and oestrogen deprivation in the management of breast cancer.

\section{Chemoprevention}

Many speakers referred to several large trials of hormone manipulation as a primary preventive strategy in high-risk groups (reviewed by Powles [7]). However, the general view was one of caution. Andrea Decensi (European Institute of Oncology, Milan, Italy) highlighted the fact that none of the studies have (yet) demonstrated any survival advantage using this approach, and Jack Cuzick (Cancer Research UK, London, UK) pointed out the relatively high number of toxic complications of therapy experienced.

\section{Pregnancy and prognosis}

Henning Mouridsen (Rigshospitalet, Copenhagen, Denmark) presented data from the Danish Cancer Registry suggesting that pregnancy has little or no effect on the prognosis of breast cancer presenting before, during or after pregnancy (with the exception that breast cancer presenting within 2 years of pregnancy carries a poor prognosis). There is, thus, little evidence to discourage women who have had breast cancer from having children.

\section{Ductal carcinoma in situ}

Staging, surgery and the role of radiotherapy for ductal carcinoma in situ were discussed. The general consensus regarding treatment was that postoperative radiotherapy significantly reduced the incidence of local recurrence, but that there was no evidence that this treatment had any significant impact on OS. The question of how to select those patients who would benefit from postoperative radiotherapy remains unanswered. Melvin Silverstein (Norris Cancer Centre, Los Angeles, CA, USA) and coworkers initially reported the Van Nuys Prognostic Index, a quantitative algorithm that used tumour size, margin width, grade and comedo-type necrosis to predict the likelihood of local recurrence following breast conservation treatment in patients with ductal carcinoma in situ [8]. At this meeting he presented an updated version of the index, which also included the patient's age as an independent factor, as a guide to assessing the patient's need for postoperative radiotherapy.

\section{Conclusion}

The consensus statement from the 8th St Gallen Breast Cancer Conference will be an important guide to the management of early breast cancer over the next 2 years. Although change is, of necessity, a slow process, this meeting offered stimulating debates, some of which will impact upon this year's statement and some of which will affect statements in years to come.

\section{Competing interests}

Both authors' attendance at this conference was made possible by an educational grant from Pharmacia Ltd. 


\section{References}

1. ATAC Trialists' Group: Anastrozole alone or in combination with tamoxifen versus tamoxifen alone for adjuvant treatment of postmenopausal women with early breast cancer: first results of the ATAC randomised trial. Lancet 2002, 359:21312139.

2. International Breast Cancer Study Group: http://www.ibcsg.org/ pps/SOFT-TEXT-PERCHE_slides-Oct02.ppt

3. Albain K, Green S, Osborne K, Cobau C, Levine E, Ingle J, Pritchard K, Schneider J, O'Sullivan J, Hess E, Martino S: Tamoxifen $(T)$ versus cyclophosphamide, adriamycin and 5 -FU plus either concurrent of sequential $\mathrm{T}$ in postmenopausal, receptor(+), node (+) breast cancer: a Southwest Oncology Group phase III intergroup trial (SWOG-8814, INT-0100) [abstract]. Proc Am Soc Clin Oncol 1997, 450:128a.

4. Henderson IC, Berry D, Demetri G, Cirrincione G, Goldstein L, Martino S, Ingle JN, Cooper MR, Canellos G, Borden E, Fleming G, Holland JF, Graziano S, Carpenter J, Muss H, Norton L: Improved disease-free and overall survival from the addition of sequential paclitaxel but not from the escalation of doxorubicin dose level in the adjuvant chemotherapy of patients with node-positive primary breast cancer [abstract]. Proc Am Soc Clin Oncol 1998, 17:101a.

5. French Adjuvant Study Group: Benefit of a high-dose epirubicin regimen in adjuvant chemotherapy for node-positive breast cancer patients with poor prognostic factors: 5-year follow-up results of French Adjuvant Study Group 05 Randomized Trial. $J$ Clin Oncol 2001, 19:602-611.

6. Citron ML, Berry DA, Cirrincione C, Hudis C, Winer EP, Gradishar WJ, Davidson NE, Martino S, Livingston R, Ingle JN, Perez EA, Carpenter J, Hurd D, Holland JF, Smith BL, Sartor Cl, Leung EH, Abrams J, Schilsky RL, Muss HB, Norton L: Randomized trial of dose-dense versus conventionally scheduled and sequential versus concurrent combination chemotherapy as postoperative adjuvant treatment of node-positive primary breast cancer: first report of intergroup trial c9741/cancer and leukemia group B trial 9741. J Clin Oncol 2003, 21:14311439.

7. Powles TJ: Anti-oestrogenic chemoprevention of breast cancer: the need to progress. Eur J Cancer 2003, 39:572-579.

8. Silverstein MJ, Lagios MD, Craig PH, Waisman JR, Lewinsky BS, Colburn WJ, Poller DN: A prognostic index for ductal carcinoma in situ of the breast. Cancer 1996, 77:2267-2274.

\section{Correspondence}

Robert J Jones, Beatson Oncology Centre, Western Infirmary of Glasgow, Glasgow G11 6NT, UK. Tel: +44 (0)141 211 2000; fax: +44 (0)1412112891; e-mail: R.Jones@beatson.gla.ac.uk 